\title{
Validation of the Secondary Predictive Model Developed for Salmonella Enteritidis SE86 Growth on Homemade Mayonnaise Salad
}

\author{
Susana Elias (I), Anderson Sant'Ana (II), Eduardo Tondo (I)
}

(I) UFRGS - Universidade Federal de Rio Grande do Sul (Avenida Bento Gonçalves, 9500 Prédio 43212 Laboratório 205 Porto Alegre/RS), (II) UNICAMP - Universidade de Campinas

(Rua Monteiro Lobato, 80 CEP 13083862 Caixa-postal 6121 Campinas/SP)

\section{Resumo}

Salmonellosis is the foodborne illness with the major incidence worldwide.

During the last decade, a specific strain Salmonella Enteritidis SE86 (SE86) has been identified as the major etiological agent responsible for salmonellosis in the State of Rio Grande do Sul, Southern Brazil, and the main food vehicle was homemade mayonnaise. Mayonnaise, often prepared with raw eggs (food vehicles of S. Enteritidis), is widely consumed and probably the most used sauce around the world. It is a food preparation frequently involved in foodborne outbreaks worldwide. Therefore this study aimed to validate a secondary predictive model developed for SE86 growth on homemade mayonnaise salad. The primary models were built by fitting data (growing curves of SE86 on homemade mayonnaise at $7,10,15,20,25,30$ and $37^{\circ} \mathrm{C}$ ) to the Baranyi's DMFit, generating $\mathrm{R}^{2}$ values greater than 0.98 . The secondary model was fitted with Ratkowsky equation, generating $\mathrm{R}^{2}$ and RMSE values of 0.99 and 0.016 , respectively. Experimental data demonstrated smaller lag phases than those generated by tertiary models (ComBase and PMP), suggesting that SE86 is well adapted for growing on mayonnaise. In addition, a questionnaire that assessed the practices adopted by homemade mayonnaise consumers and handlers was answered by 493 people that lived in Rio Grande do Sul. The most used storage practices (considering time and temperature) were used to validate the model developed in this study. First mayonnaise was prepared using the most cited recipe by 
respondents, then SE86 was inoculated on mayonnaise and it was left for 1 hour at $5^{\circ} \mathrm{C}$ (simulating the refrigerator storage), after it was left for 1 hour at $25^{\circ} \mathrm{C}$ (simulating the room temperature storage). After mayonnaise remained 24 hours at $5^{\circ} \mathrm{C}$ and finally 1 hour at $25^{\circ} \mathrm{C}$. The temperature measurement was performed every 30 minutes for 24 hours period and every 10 minutes for 1 hour periods. Sample points were collected every 1 hour during the first 4 hours and after 1 point every 4 hours for 24 hours period. For 1 hour intervals, 1 point was collected every 30 minutes. Experimental data and model simulation showed similar results for growth of SE86 on mayonnaise. In conclusion, the developed model can be used to predict the growth of Salmonella spp. on mayonnaise under various temperatures.

Palavras-Chave: Predictive Model, Salmonella, Mayonnaise, Validation Agência de Fomento: CAPES 